\title{
CARDIAC ULTRASOUND MOTION DETECTION BY ELASTIC REGISTRATION EXPLOITING TEMPORAL COHERENCE
}

\author{
María J. Ledesma-Carbayo ${ }^{\ddagger}$, Jan Kybic ${ }^{\S *}$, Michael Sühling ${ }^{\S}$, \\ Patrick Hunziker ${ }^{\natural}$, Manuel Desco ${ }^{\sharp}$, Andrés Santos ${ }^{\ddagger}$ and Michael Unser ${ }^{\S}$ \\ ‡ ETSI Telecomunicación, Universidad Politécnica de Madrid, Spain \\ email: mledesma@die.upm.es
}

\author{
$\S$ Biomedical Imaging Group, Swiss Federal Institute of Technology Lausanne, Switzerland \\ email: Michael.Unser@epfl.ch, kybic@ieee.org \\ ${ }^{\natural}$ Medical Intensive Care Unit, University Hospital, Basel, Switzerland \\ * Medicina y Cirugía Experimental, Hospital G.U. Gregorio Marañón, Madrid, Spain
}

\begin{abstract}
We propose a new global registration method for estimating the cardiac displacement field in 2D sequences of ultrasound images of the heart. The basic idea is to select a reference frame (e.g., the first image of a cycle) and to map each image in the sequence to it using elastic deformation. What makes our method specific is the use of a semi-local parametric model of the deformation (spatiotemporal spline), and the reformulation of the registration task as a global spatio-temporal optimization problem. The scale of the spline model controls the smoothness of the displacement field. Our algorithm uses a multiresolution optimization strategy for higher speed and robustness.

We validated the accuracy of our algorithm by applying it to a synthetic sequence; this one heart-cycle test sequence was generated by deforming a reference frame according to a realistic motion model and by adding random noise to it. Finally, we present results on real data from normal and pathological subjects to illustrate the clinical applicability of our method.
\end{abstract}

\section{INTRODUCTION}

Cardiac motion estimation constitutes an important aid for the quantification of the elasticity and contractility of the myocardium. Currently, the reference modality is MR imaging, especially tagged MRI, which permits to obtain

\footnotetext{
*Currently with Odyssée, INRIA, Sophia-Antipolis, France.

This work was supported in part by grant No 3200-059517.99/1 from the Swiss Science Foundation, and the Spanish project III-PRICIT Comunidad de Madrid. We would like to thank M. Arigovindan for fruitful discussions and participation in the software development.
}

dense cardiac displacement fields and derived parameters such as myocardial strain with good accuracy [1-4]. However, the imaging method most widely used to assess cardiac function is echocardiography, mostly because of its availability and noninvasiveness. The quality of cardiac ultrasound images keeps improving steadily, and there is a strong incentive to develop algorithms for the estimation of heart motion [5-7].

Most of the aforementioned approaches are based on deformable and mechanical modeling. They require a presegmentation step, which is particularly difficult in the case of cardiac ultrasound images due to the noise and the complexity of cardiac structures $[5,6]$. Speckle tracking techniques have also been proposed [7].

In this article, we propose to compute a dense cardiac displacement field using a temporal elastic registration algorithm. It optimizes a global pixel-based criterion assessing the quality of the fit over the whole sequence at once in order to find an optimal displacement field. Our algorithm can treat ultrasound sequences directly, avoiding the segmentation step. The temporal correlation of speckle (reported in [8]) is exploited for the registration, partially compensating for the relatively low quality of the ultrasound images. Controlled smoothness and temporal coherence is obtained implicitly by introducing a semilocal parametric representation of the spatio-temporal displacement field. The presented algorithm enhances our prior work in [8] (inspired by $[9,10])$ where we considered forward and backward elastic registration of consecutive image pairs and constructed the global movement from interframe displacements. As in this earlier work, we achieve reasonable speed and robustness by applying a multiresolution strategy. 
Our main interest is to apply our algorithm to 2D ultrasound sequences to estimate the motion of the heart. The main hypothesis is that the probe and patient do not move (breathhold imaging) while a sequence of $N$ images (or frames) is acquired by the operator; such a sequence must cover the cardiac cycle entirely.

The following sections describe our method in detail, and show its validity using data from both synthetic heart sequences and real patients.

\section{SPATIO-TEMPORAL ELASTIC REGISTRATION}

\subsection{Variational approach}

Given an image sequence $f(t, \mathbf{x})$, the goal is to estimate a dense displacement field $\mathbf{g}(t, \mathbf{x})$ over the whole sequence. We choose to represent the movement with respect to the first frame of the sequence: a point at coordinate $\mathbf{x}$ in the first frame $\left(t=t_{0}\right)$ will move to the location $\mathbf{g}(t, \mathbf{x})$ at time $t$. In other words, the warped sequence $f_{w}(t, \mathbf{x})=f^{c}(t, \mathbf{g}(t, \mathbf{x}))$ should not move and be at all times as similar as possible to the reference frame $f\left(t=t_{0}, \mathbf{x}\right)$. This is quantified by a criterion $E$, evaluated over the whole sequence:

$$
E=\frac{1}{\|I\| N} \sum_{k \in T} \sum_{\mathbf{i} \in I}\left(f^{c}(k, \mathbf{g}(k, \mathbf{i}))-f^{c}\left(t_{0}, \mathbf{i}\right)\right)^{2}
$$

where $f^{c}(t, \mathbf{x})$ denotes the continuously-defined version of $f(t, \mathbf{x})$. Here, we use cubic spline interpolation which has the advantage of good accuracy and also facilitates the analytical evaluation of spatial derivatives [11].

\subsection{Motion model}

The displacement field $\mathbf{g}$ is represented by a time-space separable linear model with parameters $\mathbf{d}_{\mathbf{j}, l}$ :

$$
\mathbf{g}(t, \mathbf{x})=\mathbf{x}+\sum_{l \in \mathbb{Z}} \sum_{\mathbf{j} \in \mathbb{Z}^{N}} \mathbf{d}_{\mathbf{j}, l} \phi_{\mathbf{j}}(\mathbf{x}) \psi_{l}(t)
$$

where $\phi_{\mathbf{j}}(\mathbf{x})$ influences spatial deformations for each frame and $\psi_{l}(t)$ the temporal coherence of the deformation. As shown in $[8,9]$, B-splines constitute a good choice for the spatial basis functions $\phi$. For the temporal model, some researchers have proposed using harmonic basis functions to impose temporal smoothness [1,2]. Here instead, we choose the B-splines for the temporal dimension as well, because of their computational simplicity, good approximation properties and implicit smoothness (minimum curvature property). This leads to the following expression for g:

$$
\mathbf{g}(\mathbf{x})=\mathbf{x}+\sum_{l \in \mathbb{Z}} \sum_{\mathbf{j} \in \mathbb{Z}^{N}} \mathbf{d}_{\mathbf{j}, l} \beta_{x}(\mathbf{x} / h-\mathbf{j}) \beta_{t}(t / s-l)
$$

where the basis functions $\beta_{x}(\mathbf{x})$ are placed on a rectangular grid and $\beta_{t}(t)$ at regularly spaced time points. The scale parameters $h$ and $s$ govern the knot spacing, the total number of parameters $\mathbf{d}_{\mathbf{j}, l}$, and the smoothness of the solution. The parameters used in our experiments are typically a spatial scale between control nodes of $h=32$ and a temporal gap between nodes of $s=4$. We represent the spatial and temporal deformation using quadratic or cubic splines.

We define the solution to our registration problem as a minimization $\mathbf{g}=\arg \min _{\mathbf{g} \in G} E(\mathbf{g})$, which can be solved using a multidimensional optimization algorithm. As in [8], we found that a multi-scale gradient-like optimizer was adequate for our purpose. The main idea is to proceed in a coarse-to-fine fashion on both the image sequence and motion field (spatio-temporal wavelet-like pyramids).

\subsection{Constraints}

The motion model (3) can be further constrained using prior knowledge about the motion field. First, the motion at $t=t_{0}$ (we let $t_{0}=0$ ) must be zero. This takes out one degree of freedom from our problem and leads to a modified basis function set:

$$
\psi_{l}^{\prime}(t)=\psi_{l}(t)-\frac{\psi_{l}(0) \psi_{0}(t)}{\psi_{0}(0)}
$$

Similarly, if our sequence contains a full cycle, we impose $\mathbf{g}(t)=0$ for $t=T$.

\section{VALIDATION}

This section presents our validation experiments. We compare results with our previous algorithm [8].

\subsection{Simulated Sequence Model}

Since the true cardiac motion is not available, the only way to test and evaluate the accuracy of our algorithm is to apply it to realistic, synthetically generated data. Our test sequence was generated by warping an end-diastole apical view image using cubic spline interpolation according to the following motion model

$$
\mathbf{r}_{0, i}(\mathbf{x})=\left[\begin{array}{c}
\sin ^{2}(i \pi / T) a_{x} \sin \frac{\pi\left(x_{0}-x\right)}{2\left|x_{\max }-x_{0}\right|} \\
\sin ^{2}(i \pi / T) a_{y} \frac{\left|y_{m} a x-\mathbf{y}\right|}{\left|y_{m} a x-y_{0}\right|}
\end{array}\right]^{T}
$$

where $i$ is the frame index, $y_{0}$ is the coordinate of the insertion of the mitral valve, $y_{\max }$ the coordinate of the apex and $x_{0}$ is the coordinate of the left ventricular long axis, which is oriented vertically; i.e., parallel to axis $y$. We corrupted the deformed images with multiplicative Rayleigh noise $\eta_{m}$ resembling speckle, and an additive Gaussian noise $\eta_{a}[12$, $13]$.

$$
n(\mathbf{x})=\eta_{m} \sqrt{f(\mathbf{x})}+\eta_{a}
$$



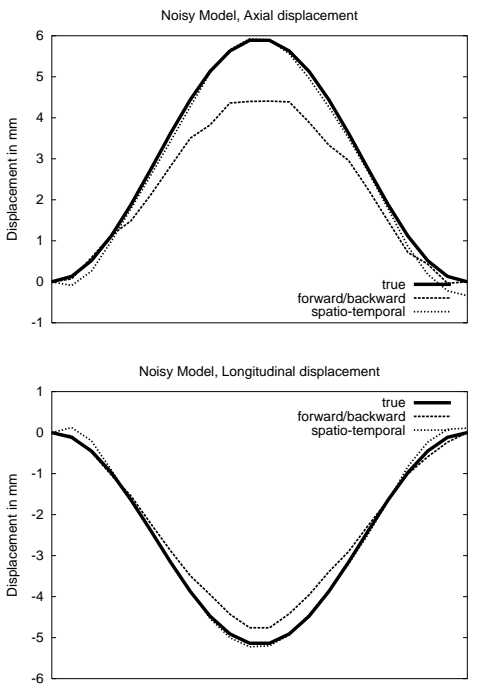

Fig. 1. Simulated sequence results. Axial (top) and longitudinal (botton) displacements in $\mathrm{mm}$ for a middle-septum point. Real displacements, results of the forward/backward registration, and the superior results of the new algorithm.

where $\eta_{a} \sim \mathcal{N}(0, \sigma), \eta_{m} \sim \mathcal{R}(\alpha)=\frac{z}{\alpha^{2}} e^{-z^{2} / 2 \alpha^{2}}$

and $f(\mathbf{x})$ is the original image with intensities in the $0 \sim$ 255 range. We generated four synthetic sequences incrementing noise ( $\sigma=10$ and $\alpha=0.4, \sigma=20$ and $\alpha=0.8$, $\sigma=40$ and $\alpha=1.6, \sigma=80$ and $\alpha=3.2$ )

\subsection{Real Sequences}

Real data from 4 normal volunteers and 4 ischemic patients were acquired with a Siemens-ACUSON Sequoia ${ }^{\circledR}$ scanner (Mountain View, CA, USA). Two and four chamber view sequences of the left ventricle were analyzed.

\section{EXPERIMENTS AND RESULTS}

\subsection{Simulated Sequence}

This section presents the results with the test sequence described in Section 3.1. First, a series of experiments was conducted to choose the most suitable parameters for the registration algorithm.

Figure 1 shows the comparison between the axial and longitudinal displacements obtained with our prior algorithm [8] (forward-backward) and the new spatio-temporal algorithm on a simulated sequence with $\sigma=20$ and $\alpha=$ 0.8 . These curves were obtained for a middle-septum point (a point in the middle of the vertical wall on the left in the images). The improvement of the new algorithm is noticeable; its motion estimates are much more accurate.

\begin{tabular}{|c||r|r|r|r|}
\hline Algorithm & $\begin{array}{r}\alpha=0.4 \\
\sigma=10\end{array}$ & $\begin{array}{r}\alpha=0.8 \\
\sigma=20\end{array}$ & $\begin{array}{r}\alpha=1.6 \\
\sigma=40\end{array}$ & $\begin{array}{l}\alpha=3.2 \\
\\
\sigma=80\end{array}$ \\
\hline Forw-Back & 0.54 & 0.79 & 1.41 & 4.70 \\
\hline Spat-Temp & 0.30 & 0.31 & 0.38 & 0.83 \\
\hline
\end{tabular}

Table 1. Mean Square Error in $\mathrm{mm}$ in 4 simulated sequences with different levels of noise using the current approach (Spat-Temp) and our prior algorithm (ForwBack) [8]

To assess quantitatively the performance and the robustness against noise, we calculated the mean square error over the whole sequence for 85 selected points within the myocardium for the four synthetic sequences. Table 1 shows the mean square error over the whole sequence compared with our previous approach [8]. Higher robustness against noise is achieved with the current approach.

\subsection{Real Sequences}

These experiments used real sequences from normal volunteers and ischemic patients. Figure 2 shows an example of the computed displacement field vectors of four-chamberview sequences of a normal volunteer and an ischemic patient. The ischemic patient presents hypokinetic function of the lateral wall and basal and distal septum segments. The displacement field correlates well with the diagnosis.
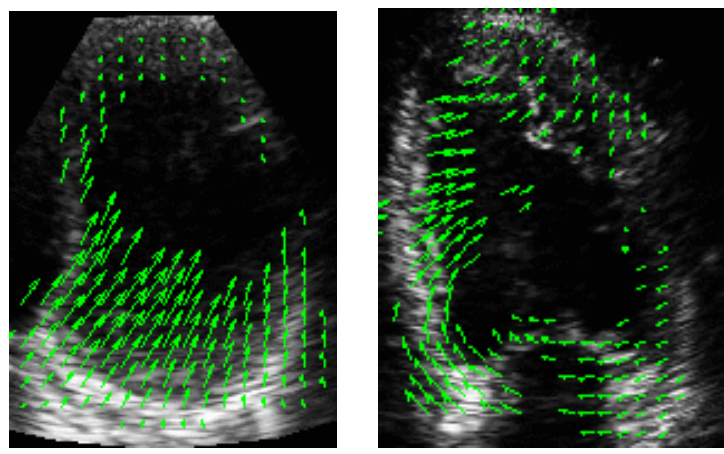

Fig. 2. Displacement field at the mechanical systole for a normal subject (left) and an ischemic patient with severe hypokinesis (right).

In Figure 3, we show the same results quantitatively for a point in the basal septum segment for the normal and ischemic cases for the contraction phase. The ischemic patient shows much smaller movement amplitude. 

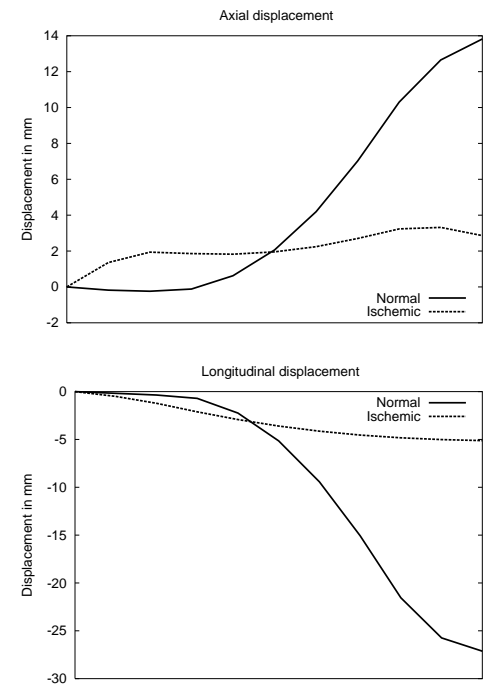

Fig. 3. Axial and longitudinal systolic displacements of a basal septum segment point for a normal volunteer and an ischemic patient with severe hypokinesis.

\section{CONCLUSIONS}

We have presented a new algorithm that performs spatiotemporal registration of ultrasound sequences. The method is fully automatic and provides motion detection exploiting temporal coherence of the movement. The method has been validated on simulated and real sequences. We have demonstrated that the proposed method is able to estimate motion accurately and that it provides plausible heart displacements and velocity fields from real echocardiograms. The results obtained with real data from normal and ischemic patients are promising and strongly suggest clinical applicability. Further clinical validation is planned for the near future.

\section{REFERENCES}

[1] P. Clarysse, C. Basset, L. Khouas, P. Croisille, D. Friboulet, C. Odet, and I. Magnin, "Two-dimensional spatial and temporal displacement and deformation field fitting from cardiac magnetic resonance tagging," Medical Image Analysis, vol. 4, no. 4, pp. 253-268, 2000.

[2] J. C. McEachen, A. Nehorai, and J. S. Duncan, "Multiframe temporal estimation of cardiac nonrigid motion," IEEE Trans. Med. Imag., vol. 9, pp. 651-664, April 2000.

[3] P. Shi, A. J. Sinusas, R. T. Constable, and J. S. Duncan, "Volumetric deformation analysis using mechanicsbased data fusion: applications in cardiac motion re- covery.," International Journal of Computer Vision, vol. 35, no. 1, pp. 87-107, 1999.

[4] J. Declerck, J. Feldmar, and N. Ayache, "Defininion of a 4D continuous planispheric transformation for the tracking and the analysis of left-ventricle motion," Medical Image Analysis, vol. 2, no. 2, pp. 197-213, 1998.

[5] C. Nastar and N. Ayache, "Frequency-based nonrigid motion analysis application to four dimensional medical images," IEEE Trans. Pattern Anal. Mach. Intell., vol. 18, pp. 1067-1079, November 1996.

[6] X. Papademetris, A. J. Sinusas, D. P. Donald, and J. S. Duncan, "Estimation of 3D left ventricular deformation from echocardiography," Medical Image Analysis, vol. 5, pp. 17-28, March 2001.

[7] F. Yeung, F. Levinson, D. Fu, and K. J. Parker, "Feature-adaptive motion tracking of ultrasound image sequences using a deformable mesh," IEEE Trans. Med. Imag., vol. 17, pp. 945-956, Dec. 1998.

[8] M. J. Ledesma-Carbayo, J. Kybic, M. Desco, A. Santos, and M. Unser, "Cardiac motion analysis from ultrasound sequences using non-rigid registration," in Proc. MICCAI 2001, Lecture Notes in Computer Science, vol. 2208 (W. J. Niessen and M. A. Viergeber, eds.), pp. 889-896, Springer Verlag, Berlin, Oct. 2001.

[9] J. Kybic, P. Thévenaz, A. Nirkko, and M. Unser, "Unwarping of unidirectionally distorted EPI images," IEEE Trans. Med. Imag., vol. 19, pp. 80-93, Feb. 2000.

[10] J. Kybic and M. Unser, "Multidimensional elastic registration of images using splines," in Proceedings of ICIP, (Vancouver, Canada), 2000.

[11] M. Unser, "Splines: A perfect fit for signal and image processing," IEEE Signal Processing Magazine, vol. 16, pp. 22-38, Nov 1999.

[12] X. Zong, A. Laine, and E. Geiser, "Speckle reduction and contrast enhancement of echocardiograms via multiscale nonlinear processing," IEEE Trans. Med. Imag., vol. 17, no. 4, pp. 532-540, 1998.

[13] T. Loupas, W. McDicken, and P. Allan, "An adaptive weighted median filter for speckle suppression in medical ultrasonic images," IEEE Trans. Circ. Syst., vol. 36, pp. 129-135, Jan. 1989. 